\title{
Metastatic Renal Cell Carcinoma Presenting as Breast Mass in a Male
}

\author{
Jaideep Das Gupta ${ }^{1}$, Indranil Sen ${ }^{1}$, SP Chowdhury ${ }^{1}$, Anjan Das ${ }^{2}$ \\ Departments of ${ }^{1}$ General Medicine and ${ }^{2}$ Pathology, Calcutta National Medical College, Kolkata, West Bengal, India.
}

\begin{abstract}
Corresponding Author:
Dr. Jaideep Das Gupta

Email: jaydocgupta@gmail.com

This is an Open Access article distributed under the terms of the Creative Commons Attribution License (creativecommons.org/ licenses/by/3.0).
\end{abstract}

Received Accepted

Published

August 31, 2017

January 4, 2018

January 20, 2018

\begin{abstract}
Background: Metastasis to breast in males from renal cell carcinoma is a very rare occurrence. This subject is of importance since it requires a different approach in prognosis and management from primary breast cancer. Historically, the prognosis for such a patient has been extremely poor. Case Report: We hereby report a 60 year old male patient with renal cell carcinoma metastasizing to the breast and lungs. Conclusion: This case report will add to the ever-expanding understanding of the complexities of cancer presentation and its management.
\end{abstract}

Keywords: Breast, Breast Neoplasms, Male, Renal Cell Carcinoma, Prognosis.

\section{Introduction}

Breast cancer in males is extremely rare, representing $<1 \%$ of all cancers in males with an approximate incidence of 1 case per 100,000 men. Metastases to the breast from other organs are not common; the prevalence of such lesions ranges from 1.7 to $6.6 \%$ [1]. The most common sources of extra-mammary metastases to the breast are lymphomas/leukemias and melanomas. Some of the less common sources include carcinomas of the lung, ovary, and stomach, and infrequently, carcinoid tumors, hyper-nephromas, carcinomas of the liver, tonsil, pleura, pancreas, cervix, perineum, endometrium and bladder [2]. Only in three percent of the cases is the primitive tumor of renal origin. On the other hand renal cell carcinoma accounts for $2-3 \%$ of all malignancies with 40 to $50 \%$ cases developing distant metastasis [3]; it is the $7^{\text {th }}$ most common malignancy in men [4]. Male: female ratio ranges from 1.5:1 to 2.5:1 worldwide [5]. But in India the ratio is around 4:1 [6]. Although RCC can metastasize to unusual sites, the commonly favoured include the lung, bone, liver, abdomen, and the retro-peritoneum in decreasing order of frequency [7].
To our knowledge till date there are very few published cases in the literature of metastatic RCC to the breast in a male [8]. Herein we report a case of renal cell carcinoma in a male who was incidentally diagnosed to have metastatic breast cancer during work up.

\section{Case Report}

A 60 year old man presented to our emergency department with complaints of sudden onset urinary retention and a gradually increasing pain localized to the lower abdomen for one day. He gave a history of respiratory distress for one week which was insidious in onset, gradually progressive and increased on supine posture. He also gave history of dysuria since past one week which was associated with decreased urine output. He gave constitutional symptoms of generalized weakness, loss of appetite since past one month. He denied any symptoms of fever, chest pain, cough, sputum production, hematuria, pain abdomen, mass abdomen, nausea, or swelling of feet. His past history was significant for an episode of ischemic cerebrovascular accident six years back for which he was treated conservatively and there was no 
residual neurological deficit. His family history was insignificant and he was a teetotaller.

On examination he was restless, with irregular pulse, tachypnea, severe pallor and suprapubic tenderness with swelling. His blood pressure was $140 / 90 \mathrm{mmHg}$, temp $38.6^{\circ} \mathrm{C}$ and his general appearance was cachectic. He was immediately catheterised and subsequently relieved of his pain after passing large volume of urine. Respiratory examination revealed decreased breath sounds over whole of left lung and moderate crackles in the right lower lobe. Other system examination was normal. On physical examination, there was a nontender hard mass approximately $2 \mathrm{~cm}$ in diameter not fixed to any surrounding structures in the upper outer quadrant of the right breast, there was no superficial lymphadenopathy. The ultrasonographic examination showed the heterogeneous space

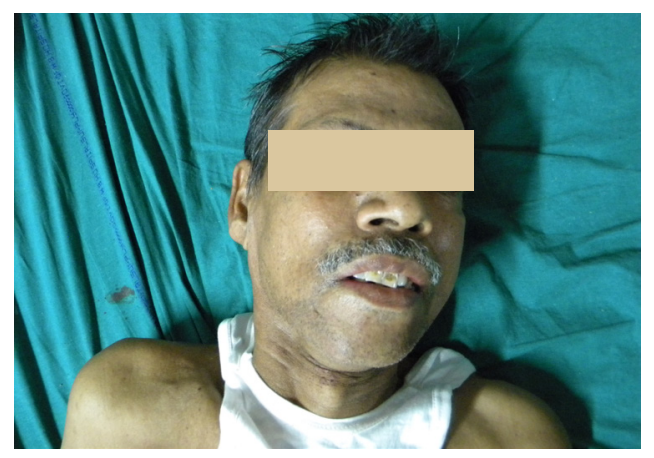

Fig.1: Patient image.

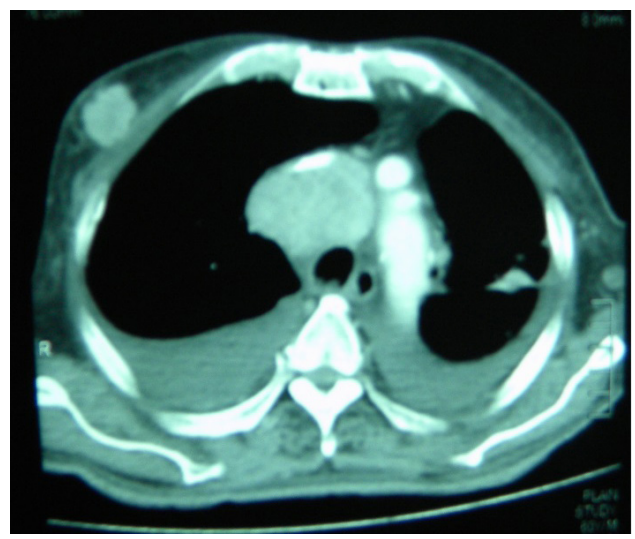

Fig.2: CT scan of thorax showing irregular heterogenous enhancing, soft tissue attenuating mass lesion in the anterior chest wall in right upper thorax. occupying lesion with a maximum diameter of $19 \times 16 \mathrm{~mm}$ and irregular margins in twelve o clock position of right breast. The contrast enhanced CT scan of thorax reported similar findings with enlarged lymph nodes in the superior mediastinum, pre-tracheal, carinal and right hilar region, further near collapse of left lower lobe with sub-segmental collapse of left upper lobe and multifocal pulmonary nodules scattered bilaterally throughout both lung parenchyma was noted suggesting pulmonary secondaries. Fine needle aspiration of the breast mass revealed the presence of a solid nest of cells with clear cytoplasm and small, round hyperchromatic nuclei surrounded by a rich vascular network suggestive of metastatic renal cell cancer.

His initial blood tests revealed the following: hemoglobin: $5.8 \mathrm{~g} / \mathrm{dL}$, total leukocyte count: $17.3 \times 10^{9} / \mathrm{L}$ and platelets: $319 \times 10^{9} / \mathrm{L}$. Electrolytes and urea were as follows: $\mathrm{Na} 130 \mathrm{mmol} / \mathrm{L}, \mathrm{K}^{+} 4.2$ $\mathrm{mmol} / \mathrm{L}$, urea $20 \mathrm{~m} / \mathrm{dL}$ and creatinine $1.34 \mathrm{~m} / \mathrm{dL}$. His urine examination revealed traces of blood
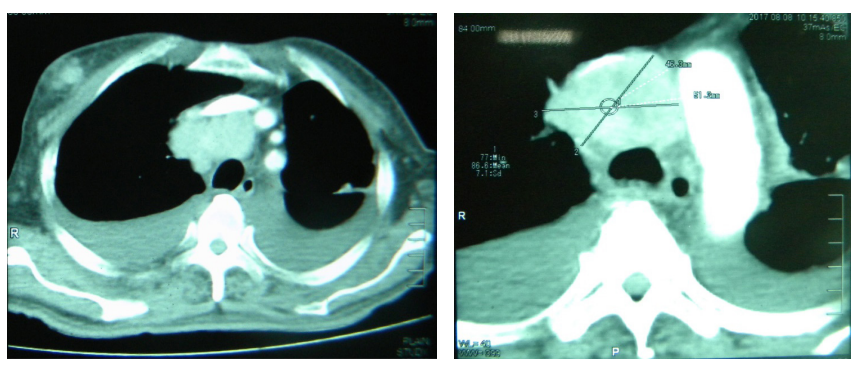

Fig.3,4: CT scan of thorax showing moderate pleural effusion with enlarged discrete lymph nodes in the superior mediastinum.
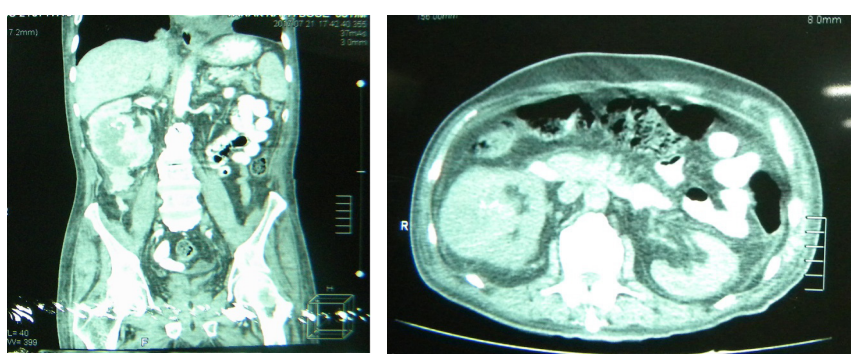

Fig.5,6: CT scan of abdomen showing large irregular outlined heterogeneously enhancing soft tissue density mass lesion involving the posterolateral aspects of mid and lower pole of right kidney. 


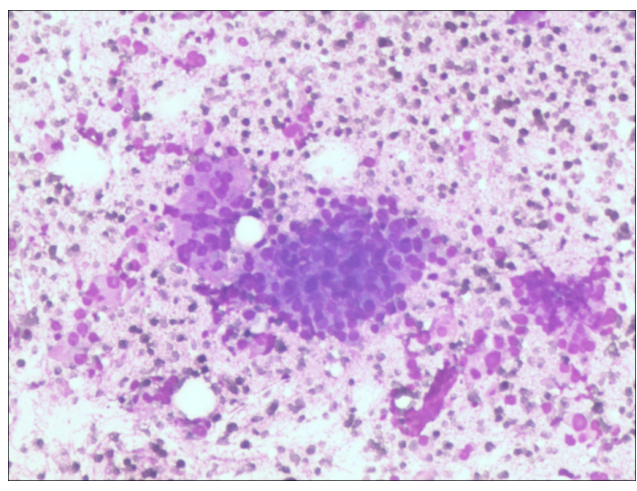

Fig.7: Photomicrograph showing cytology of breast mass $(10 \times L \& G)$.

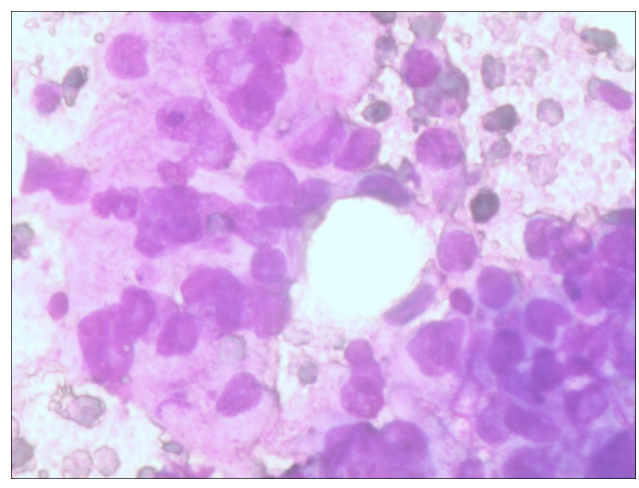

Fig.8: High power view of the photomicrograph of breast mass.

and protein, 8-10 pus cells. Liver function tests (LFTs) were within normal limits except for serum albumin $2.5 \mathrm{~g} / \mathrm{dL}$. Chest radiography demonstrated multiple nodules of varying sizes spread over both lungs and mild bilateral pleural effusion. The electrocardiogram showed irregularly irregular heart rhythm with absent $\mathrm{P}$ waves suggestive of atrial fibrillation.

The patient had an ultrasound of the abdomen which showed a heterogeneous space occupying lesion of size $7.3 \times 7.1 \mathrm{~cm}$ in the lower pole of right kidney. His subsequent contrast enhanced CT scan of abdomen showed a large irregular outlined heterogeneously enhancing soft tissue mass about $79 \times 62 \mathrm{~mm}$ involving the postero-lateral aspects of mid and lower pole of right kidney. It revealed internal hypo-dense areas suggestive of necrosis and small calcified foci. A

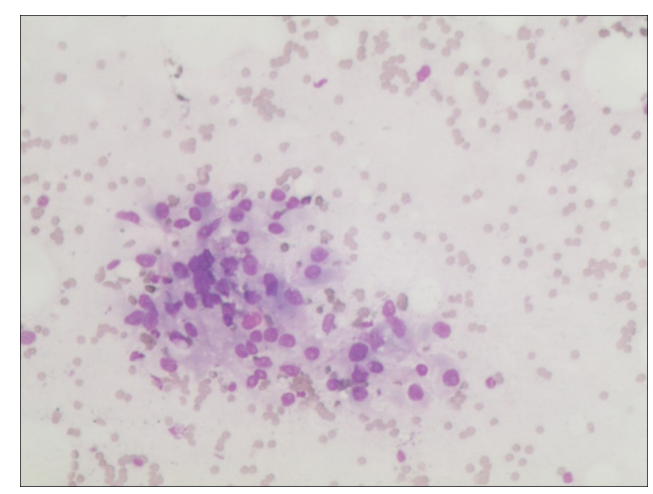

Fig.9: Photomicrograph showing cytology of renal mass.

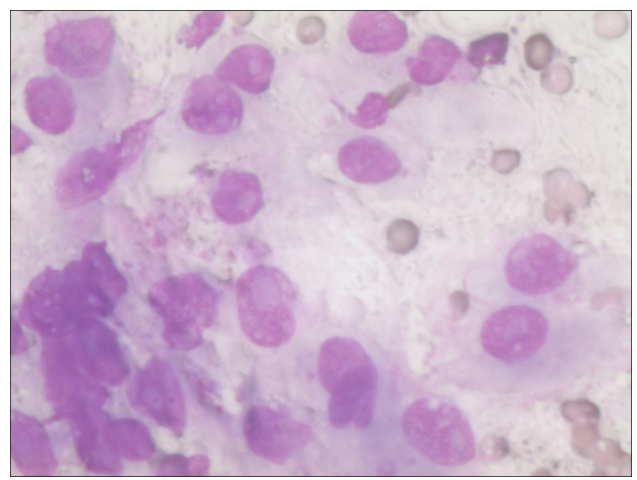

Fig.10: High power view of renal mass showing malignant cells.

biopsy was taken from the mass histopathological examination of which was consistent with the findings of high grade renal cell carcinoma. Thus the final impression is of a renal cell carcinoma metastasizing to the breast and lungs.

Due to the progression and the extension of the disease, Pazopanib (Votrient), a vascular endothelial growth factor inhibitor (VEGFI), was administered orally in the dose of $400 \mathrm{mg}$ twice a day in consultation with our oncology department.

\section{Discussion}

Although initial presentation and examination findings in this case were not strongly indicative of renal cell carcinoma, further investigation in the examination illuminated the occult disease process of renal cell carcinoma. The primitive neoplasms 
that most frequently metastasize to the breast (after having excluded the case of the contralateral breast and the lymphoma) are in order of frequency; malignant melanoma, reticulosarcoma, lung carcinoma (especially in the oat-cell varieties) and, for the male sex, prostate carcinoma. Renal cell carcinomas are known for their tendency for early metastasis, and symptoms due to the metastatic lesion may be the only initial manifestation. In one study, RCC was the most common tumor found to coexist with other malignancies [9]. Renal clear cell carcinoma may metastasize when the diameter of the primary tumor exceeds $3 \mathrm{~cm}$. Because of the slow growth at the initial stage in about $60 \%$ of the cases, the primary lesion receives little attention until symptoms occur because of metastasis [10].

The original classic triad of flank pain, hematuria, and palpable mass occurs in only about $10 \%$ of patients, and palpable mass occurs in only about $10 \%$ of those patients. However, intermittent microscopic hematuria occurs in $90 \%$ of patients [11]. Clear cell RCC accounts for $\sim 85 \%$ of renal cell tumors; the other subtypes are typically summarized under the term non-clear cell RCC they include papillary, chromophobe, and Bellini duct (collecting duct) tumors. Collecting duct carcinoma constitutes fewer than $1 \%$ of kidney cancer cases. Medullary renal carcinoma is a variant of collecting duct renal carcinoma and was described initially as occurring in patients who are sickle cell traitpositive. Certain genetic conditions are associated with an increased incidence of RCC, including von Hippel-Lindau disease, hereditary papillary renal cancer, and, possibly, tuberous sclerosis [12]. Other suggested risk factors include cigarette smoking; obesity; diuretic use; exposure to petroleum products, chlorinated solvents, cadmium, lead, asbestos, and ionizing radiation; high-protein diets; hypertension; kidney transplantation; and HIV infection. Since symptoms of metastatic tumors often precede those of a primary in renal cancer, a mass in the breast must be diagnosed with this point in mind, even in the absence of a past history of renal cancer.

The treatment of renal cell carcinoma (RCC) has changed greatly over the past 15 years. Progress in the surgical management of the primary tumor and increased understanding of the molecular biology and genomics of the disease have led to the development of new therapeutic agents. The management of the primary tumor has changed owing to the realization that clean margins around the primary lesion are sufficient to prevent local recurrence, as well as the development of more sophisticated tools and techniques that increase the safety of partial nephrectomy. The management of advanced disease has altered even more dramatically as a result of new agents that target the tumor vasculature or that attenuate the activation of intracellular oncogenic pathways. If detected early, RCC is curable by surgery although a minority are at risk of recurrence. Surgical treatment is the only curative therapy for localized RCC. Radical nephrectomy remains the mainstay of surgical management, but techniques are being modified. These modifications include partial nephrectomy and resection of vena caval thrombi. In highly selected cases, surgical resection of locally recurrent RCC or of disease at a solitary metastatic site is associated with long-term survival. To treat metastatic renal cell carcinomas, targeted therapy instead of immunotherapy is the first-line therapy followed by surgical resection. Targeting agents are also superior to immunotherapy in prolonging survival [13]. Several targeting agents, such as sunitinib, temsirolimus, and sorafenib, have been used to treat patients with metastatic renal cancer. In the context of metastatic disease, a nephrectomy may still be indicated for symptomatic relief for example, severe pain or hematuria; in a small number of cases $(\approx 0.3 \%)$, regression of metastases has been reported after resection of the primary tumor. Cytoreductive nephrectomy has been shown to offer a survival benefit in selected patients with metastatic disease [14]. 


\section{Conclusion}

The case described here is unusual because the primary tumor had not declared its presence and also because of its unusual site. Because of the limited number of cases that occur and availability of follow-up information, additional studies are needed to further improve our understanding of these metastasis and give these patients an appropriate treatment. When a patient presents with a mass in the pectoral region, the possibility of metastasis should always be considered especially from the renal system. Observations in the case report described by us may not be groundbreaking, but they will nevertheless add to the everexpanding understanding of the complexities of cancer presentation and its management.

Contributors: JDG: Manuscript writing, case management and literature search; IS: Conception and design of the work; SPC: Manuscript editing, intellectual inputs and funding; AD: Histopathology and manuscript editing. JDG will act as guarantor. All authors approved the final version of this article.

Funding: None; Competing interests: None stated.

\section{References}

1. Toombs BD, Kalisher L. Metastatic disease to the breast: Clinical, pathological and radiographic features. AJR. 1977;129:673.

2. Bohman LG, Bassett LW, Gold RH, Voet R. Breast metastases from extramammary malignancies. Radiology. 1982;144:309.

3. Matveev VB, Gurarii LL, Began-Bogatskii KM. Surgical treatment of late metastases of kidney cancer. Urol Nefrol (Mosk). 1999;2:51-52.
4. International Agency for Research on Cancer. The GLOBOCAN project: cancer incidence and mortality worldwide in 2008. Available at: http://globocan.iarc.fr/ Accessed July 6, 2011.

5. Lindblad P. Epidemiology of renal cell carcinoma. Scand J Surg. 2004;93:88-96.

6. Sivaramakrishna B, Gupta NP, Wadhwa P, Hemal AK, Dogra PN, Seth A, et al. Pattern of metastases in renal cell carcinoma: A single institution study. Indian J Cancer. 2005;42:173-177.

7. Hafez KS, Novick AC, Campbell SC. Patterns of tumor recurrence and guidelines for followup after nephron sparing surgery for sporadic renal cell carcinoma. J Urol. 1997;157:2067-2070.

8. Saluja K, Thakral B, Bit-Ivan E, Kaufman M, Liu L. Fine-needle aspiration of metastatic renal cell carcinoma to a male breast: A rare initial presentation. Cyto Journal. 2014;11:8. doi:10.4103/1742-6413.131737.

9. Richardson J, Katamaya I. Neoplasm to neoplasm metastasis. Arch Path. 1971;91:135-139.

10. Lokich JJ, Harrison JH. Renal cell carcinoma: natural history and chemotherapeutic experience. J Urol. 1975;114:371-374.

11. Skinner DG, Colvin RB, Vermillion CD, Pfister RC, Leadbetter WF. Diagnosis and management of renal cell carcinoma. A clinical and pathologic study of 309 cases. Cancer. 1971;28:1165-1177.

12. Bjornsson J, Short MP, Kwiatkowski DJ, Henske EP. Tuberous sclerosis-associated renal cell carcinoma: clinical, pathological, and genetic features. Am J Pathol. 1996;149:1201-1208.

13. MotzerRJ, HutsonTE, TomczakP, Michaelson MD, Bukowski RM, Rixe O, et al. Sunitinib versus interferon alfa in metastatic renal-cell carcinoma N Engl J Med. 2007;356:115-124.

14. Kassouf W, Sanchez-Ortiz R, Tamboli P, Jonasch E, Merchant MM, Spiess pe, et al. Cytoreductive nephrectomy for T4NxM1 renal cell carcinoma: the M. D. Anderson Cancer Center experience. Urology. 2007; 69:835-838. 